Recepción: 19 / 11 / 2018

Aceptación: $28 / 12$ / 2018

Publicación: 20 / 02 / 2019

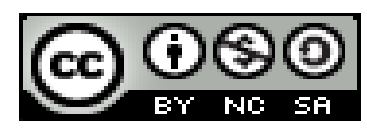

Ciencias de la salud

Artículo de Revisión

\title{
Factores que inciden en la presencia de la depresion en adolescentes
}

\author{
Factors that affect the presence of depression in adolescents
}

\section{Fatores que afetam a presença de depressão em adolescentes}

\author{
Carlos A. Borja-Delgado ${ }^{\mathrm{I}}$ \\ alex2254cabd@outlook.com \\ Estefania C. Ruilova-Mera ${ }^{\text {II }}$ \\ stefkroly-rm@live.com \\ Daisy M. Garcia-Intriago III \\ daisymonica2003@yahoo.com \\ Shirley A. Sanchez-Sanchez IV \\ shirley.sanchezs@utm.edu.ec \\ Katerine E. Guevara-Suárez V \\ katyguevara.280291@gmail.com \\ Andrés P. Morales-Tipan VI \\ andres_pmt@hotmail.com
}

Correspondencia: alex2254cabd@outlook.com

I. Médico; Hospital Eugenio Espejo; Área de Urología, Quito, Ecuador.

II. Médico Cirujano; Médico General en funciones hospitalarias; Hospital IESS; Manta, Ecuador.

III. Magister en Gerencia de Salud para el Desarrollo Local; Especialista en Gerencia y Planificación Estratégica en

Salud; Diploma Superior en Desarrollo Local y Salud; Licenciada en Enfermería; Docente de la Universidad Técnica de Manabí; Portoviejo, Ecuador.

IV. Magister en Gerencia en Salud para el Desarrollo Local; Especialista en Gerencia y Planificación Estratégica de Salud; Licenciada en Enfermería; Enfermera del Centro de Salud Portoviejo-Distrito 13D0; Docente de la Universidad Técnica de Manabí; Portoviejo, Ecuador.

v. Médica; Hospital General Ibarra; Servicio de Emergencias Pediátricas; Ibarra, Ecuador.

VI. Médico; Hospital IESS Ibarra; Área de Traumatología; Ibarra, Ecuador. 


\section{Resumen}

Las exposiciones psicosociales generales de la psicopatología del florecimiento afirman que el marco familiar desempeña un papel importante en la gestación del funcionamiento adaptativo y desadaptativo de los niños. Además, prácticamente todas las tesis de la depresión afirman que las relaciones defectuosas entre padres e hijos desempeñan un papel importante en la etiología de este perjuicio. De acuerdo con estas formulaciones teóricas, los hacedores de riesgo familiar han sido el foco de la mayoría de las indagaciones sobre la depresión en la adolescencia. Varios estudios han evidenciado que el afecto inseguro y la crianza de los hijos caracterizados por la frialdad, el rechazo, la disciplina severa y el talante poco conveniente se relacionan efectivamente con los indicios depresivos de los adolescentes. Algunas búsquedas indican que la paternidad facultada, conceptualizada como una fusión de calidez, aprobación, control firme y disciplina democrática, se asocia con las señales menos depresivas entre los adolescentes. Los factores patogénicos en el ámbito familiar, como la depresión de los procreadores, los cambios en la técnica familiar, la transgresión o el abandono, incluso pueden contribuir a la depresión en la adolescencia. Una relación causal entre desfile defectuoso y depresión es probablemente la interacción entre aspectos ambientales hereditarios, cognitivos, emocionales, interpersonales y parientes.

Palabras claves: Depresión; Adolescencia; Entorno familiar.

\section{Abstract}

The general psychosocial expositions of the psychopathology of flowering affirm that the family framework plays an important role in the gestation of the adaptive and maladaptive functioning of children. In addition, virtually all theories of depression claim that defective relationships between parents and children play an important role in the etiology of this damage. According to these theoretical formulations, family risk makers have been the focus of most of the research on adolescent depression. Several studies have shown that insecure affection and the upbringing of children characterized by coldness, rejection, severe discipline and unpleasant mood are effectively related to the depressive symptoms of adolescents. Some searches indicate that empowered paternity, conceptualized as a fusion of warmth, approval, firm control and democratic discipline, is associated with the least depressive signs among adolescents. Pathogenic factors in the family, such as the depression of procreators, changes in family technique, transgression or abandonment, can even contribute to depression in adolescence. A causal relationship between faulty parade and 
depression is probably the interaction between hereditary, cognitive, emotional, interpersonal and kinship environmental aspects.

Keys words: Depression; Adolescence; Familiar surroundings.

\section{Resumo.}

As exposições psicossociais gerais da psicopatologia da floração afirmam que a estrutura familiar desempenha um papel importante na gestação do funcionamento adaptativo e mal-adaptativo das crianças. Além disso, virtualmente todas as teorias da depressão afirmam que relacionamentos defeituosos entre pais e filhos desempenham um papel importante na etiologia desse dano. De acordo com essas formulações teóricas, os criadores de risco familiar têm sido o foco da maioria das pesquisas sobre depressão adolescente. Vários estudos demonstraram que o carinho inseguro e a educação de crianças caracterizadas por frieza, rejeição, disciplina severa e humor desagradável estão efetivamente relacionados aos sintomas depressivos dos adolescentes. Algumas pesquisas indicam que a paternidade fortalecida, conceituada como uma fusão de calor humano, aprovação, controle firme e disciplina democrática, está associada aos sinais menos depressivos entre os adolescentes. Fatores patogênicos na família, como a depressão de procriadores, alterações na técnica familiar, transgressão ou abandono, podem até contribuir para a depressão na adolescência. Uma relação causal entre parada defeituosa e depressão é provavelmente a interação entre aspectos ambientais hereditários, cognitivos, emocionais, interpessoais e de parentesco.

Palavras chaves: Depressão; Adolescência; Entorno familia.

\section{Introducción.}

La mala salud mental es el mayor problema de salud pública en los países de altos ingresos, el segundo mayor contribuyente en años vividos con discapacidades en el mundo y el problema número uno entre los niños de 10 a 14 años en Canadá y los Estados Unidos (Vos, Flaxman, Naghavi, \& Lozano, 2012). Con mayor frecuencia, se encuentra que las niñas tienen un mayor riesgo de síntomas depresivos que los niños, aunque también se ha encontrado lo contrario. Por ejemplo, en Suecia, los síntomas depresivos auto informados han aumentado entre los jóvenes de 
ambos sexos en las últimas dos décadas, además, la prevalencia de adolescentes tratados por depresión ha aumentado. Esto es especialmente preocupante dado que la depresión en la adolescencia, incluida la depresión subclínica, es un factor de riesgo para una depresión clínica más adelante en la vida, así como para otros tipos de enfermedades mentales, abuso de sustancias, suicidio, bajo nivel educativo y desempleo.

La mayoría de los problemas de salud son más comunes entre los grupos con un estatus socioeconómico más bajo, Por lo tanto, se puede esperar que los factores sociales y económicos, junto con los factores psicosociales y genéticos, desempeñen un papel en el desarrollo de los síntomas de la enfermedad mental en los adolescentes. La exposición a condiciones sociales y materiales familiares desfavorables, como el bajo nivel educativo, el desempleo, la falta de recursos y la falta de tiempo, puede plantearse como hipótesis tanto para aumentar la angustia mental entre los niños como para reducir las posibilidades de los padres de pagar, exigir y recibir una buena atención médica para sus hijos.

Una forma frecuentemente utilizada para representar las diferencias en la salud es la situación socioeconómica de un individuo, o, en el caso de los niños, el ingreso, la ocupación y la educación. La relación entre la situación socioeconómica y los síntomas depresivos no es directa, como lo demuestran algunos estudios realizados entre adolescentes y adultos (Miech \& Caspi, 1999). La comprensión del campo se ve obstaculizada por la heterogeneidad de los indicadores utilizados para definir es status socioeconómico (por ejemplo, ingresos, educación y clase ocupacional), la gravedad de la enfermedad y las definiciones de los síntomas depresivos (incluida la depresión clínica y auto informada), así como por el ajuste diferencial de factores confusos. Con respecto a los estudios en adolescentes, la mayoría encontró al menos un indicador socioeconómico bajo (según la información de los padres o la familia), vinculado al riesgo de síntomas depresivos, pero en algunos 
casos se encontró una asociación opuesta para otro indicador socioeconómico. Sin embargo, los estudios que emplean varias medidas son raros, y el empleo de medidas no refinadas, por ejemplo. exposiciones dicotomizadas, es común. Además, muchos de los estudios que muestran asociaciones entre los síntomas depresivos en la adolescencia y el ingreso o educación de los padres utilizaron un diseño transversal, generando problemas de causalidad inversa.

Otros factores sociales en la infancia que se correlacionan con los síntomas depresivos en la adolescencia o más tarde en la vida son, por ejemplo, el origen geográfico de los padres, dificultades financieras, desempleo de los padres y vivir con una sola madre. Raras veces se han estudiado las diferencias de género en la asociación entre el aspecto socioeconómico de los padres y los síntomas depresivos en la adolescencia, aunque (Reiss, 2013) concluye en una reciente revisión sistemática que existe una inconsistencia en los patrones de género en situaciones socioeconómicas y problemas de salud mental.

Poco se sabe acerca de las diferencias sociales en el riesgo de síntomas depresivos entre los adolescentes en forma de estados de bienestar típicos, donde es probable que las diferencias sociales sean menos pronunciadas. De hecho, la mayoría de los estudios se realizaron en los EE. UU., y algunos en diferentes países europeos, entre ellos España, Hungría, Holanda y el Reino Unido. Estos mostraron que la ocupación manual de los padres, pero no el bajo ingreso de los padres, se asoció con síntomas depresivos autoevaluados entre los adolescentes.

Los estudios sobre el estado social y los síntomas depresivos en la adolescencia contribuirían de manera importante a ampliar el conocimiento limitado sobre las desigualdades sociales en salud mental en este grupo de edad. Esto es especialmente importante debido al aumento en los síntomas depresivos reportados por este grupo. Por lo tanto, el objetivo fue investigar si las medidas múltiples 
Carlos A. Borja-Delgado; Estefania C. Ruilova-Mera; Daisy M. Garcia-Intriago; Katerine E. Guevara-Suárez; Andrés P. Morales-Tipan

del bajo estatus social de la familia están asociadas longitudinalmente con los síntomas depresivos en la adolescencia. Un objetivo secundario fue explorar la posible modificación del efecto de género.

\section{Metodología.}

La revisión se ha centrado en textos, documentos y artículos científicos publicados disponibles en la web, considerando que aquella herencia de la globalización nos permite acceder a mayor y mejor información a través de las herramientas tecnológicas. Los criterios de inclusión se basaron en publicaciones del habla hispana e inglesa y visible en bases de datos que nos aportaron en la historia y evolución de investigación. El motor de búsqueda ha sido herramientas académicas de la web que direccionan específicamente a archivos con validez y reconocimiento científico, descartando toda información no confirmada o sin las respectivas referencias bibliográficas.

\section{Resultados.}

Un factor de riesgo es cualquier circunstancia o situación que aumenta la probabilidad de desarrollar una enfermedad o la probabilidad de que ocurra un evento adverso. Los factores de riesgo no son necesariamente causas, sino que están asociados con el evento, y como tienen un valor predictivo, pueden usarse para la prevención (Argimón Pallás \& Jiménez Villa, 2000). La depresión en niños y adolescentes es una enfermedad compleja que tiene múltiples factores de riesgo, que ocasionalmente interactúan y puede tener un efecto acumulativo.

Es poco probable que un solo factor de riesgo pueda explicar el desarrollo de la depresión o reducir la probabilidad de ocurrencia o que controlar ese factor sea suficiente para prevenir la depresión. 
Factores que inciden en la presencia de la depresion en adolescentes

Entre los principales factores de riesgos que inciden en la aparición de la depresión en edades adolescentes se encuentran:

\section{Factores familiares y ambientales}

Los factores familiares y el contexto social podrían jugar un papel importante en el desarrollo de la depresión; básicamente aquellos factores que están más allá del control propio, que ocurren como un evento impredecible en el entorno diario y que se repiten con el tiempo.

\section{Riesgos familiares}

La depresión en los padres se considera un factor de riesgo importante, y se asocia con la depresión en los descendientes de los padres (Garber, 2006). Se ha visto que los hijos de padres con depresión muestran una probabilidad de tres a cuatro veces mayor del desarrollo de trastornos del estado de ánimo y específicamente la psicopatología materna se considera un predictor de depresión en los niños. El alcoholismo familiar también se ha asociado con una mayor probabilidad de depresión.

El contexto familiar en el que vive un niño o adolescente parece jugar un papel importante en el desarrollo de la depresión. Los factores de riesgo más comunes son la existencia de conflictos conyugales o dificultades emocionales entre uno de los padres y el niño. Otros factores de riesgo asociados con la depresión también incluyen diferentes formas de abuso, como abuso físico, emocional y sexual y negligencia en la atención, así como eventos negativos de la vida, divorcio o separación conflictiva de los padres, la pérdida de amistades y la muerte de un familiar o amigo.

Los padres que trabajan fuera del hogar, de bajos ingresos o que viven en áreas desfavorecidas (si ocurren por separado) no parecen presentar una asociación fuerte con el desarrollo de la depresión en niños y adolescentes. 
Carlos A. Borja-Delgado; Estefania C. Ruilova-Mera; Daisy M. Garcia-Intriago; Katerine E. Guevara-Suárez; Andrés

Riesgos relacionados con el medio ambiente

La depresión adolescente a menudo se asocia con la existencia de conflictos interpersonales y la existencia de rechazo por parte de diferentes miembros del entorno social, lo que aumenta los problemas de relación social. Por lo tanto, los niños y adolescentes con pocos amigos muestran una mayor probabilidad de desarrollar depresión, así como trastornos de conducta y mayor aislamiento social.

Otros factores asociados con un mayor número de síntomas depresivos incluyen vivir en estructuras familiares que son diferentes a las de los padres biológicos, problemas de salud en los adolescentes o una mala adaptación a la familia, los amigos, la escuela, el trabajo y un compañero. No parece haber una asociación con vivir en un entorno rural o urbano (Haavisto, 2004). La intimidación y la humillación (como el tratamiento degradante, reírse de los demás o sentirse ignorado) también son factores de riesgo de la depresión.

Los niños y adolescentes sin hogar, los que ingresan en albergues, los refugiados y los que tienen antecedentes de delincuencia, especialmente los que están encerrados en instituciones de seguridad, deben tener un alto riesgo de presentar trastornos mentales.

Finalmente, la adicción a la nicotina, el abuso del alcohol o el consumo de drogas ilegales también se consideran factores de riesgo asociados con la depresión.

Factores individuales

Sexo 
Antes de la adolescencia, los trastornos depresivos son prácticamente iguales en niños y niñas. Sin embargo, en la primera mitad de la adolescencia, estos trastornos son de dos a tres veces más frecuentes en las mujeres. Las posibles explicaciones incluyen los cambios hormonales que ocurren, un aumento del estrés y una mala respuesta, las diferencias en las relaciones interpersonales y una tendencia a los pensamientos intrusivos.

\section{Factores de riesgo genéticos y bioquímicos}

Entre el 20\% y el 50\% de los niños y adolescentes con trastornos depresivos presentan antecedentes familiares de depresión u otra enfermedad mental. Sin embargo, la información actual sugiere que los factores de riesgo genéticos podrían ser menos importantes en la aparición de depresión en la niñez que en la adolescencia.

Sin embargo, el peso de los factores genéticos y ambientales en el desarrollo de la depresión no está claro. Por lo tanto, los estudios realizados en gemelos indican que los síntomas depresivos podrían explicarse por la presencia de factores genéticos en el 40-70\% de los casos (Bragado, Bersabé, \& Carrasco, 1999). Sin embargo, los mecanismos de acción de los genes en diferentes niveles hasta la manifestación clínica de la depresión aún son desconocidos. Para algunos autores, cuanto más grave es la depresión, mayor es la influencia del medio ambiente, mientras que para otros, los síntomas clínicos más graves de la depresión mayor están más influenciados por la genética.

Existe alguna evidencia que indica que las alteraciones de los sistemas corticales serotoninérgicos y suprarrenales podrían estar implicadas en la biología de la depresión en niños y adolescentes. Por lo tanto, se han observado alteraciones de la función serotonérgica en niños con antecedentes familiares de depresión. Además, el aumento de los niveles de cortisol y dehidroepiandrosterona predice el inicio de la depresión en subgrupos de adolescentes con un alto riesgo psicológico de 
Carlos A. Borja-Delgado; Estefania C. Ruilova-Mera; Daisy M. Garcia-Intriago; Katerine E. Guevara-Suárez; Andrés

aparición de depresión (Health., 2005). También parece haber alteraciones en los niveles de hormona del crecimiento, prolactina y cortisol en los jóvenes quienes corren riesgo de depresión.

\section{Factores psicológicos}

Se ha pensado que el temperamento tiene una base genética / biológica, aunque la experiencia y el aprendizaje (en particular dentro del contexto social) pueden influir en el desarrollo y la expresión del mismo. La afectividad negativa se refiere a la tendencia a experimentar estados emocionales negativos, que se acompañan de características de comportamiento (como la inhibición social y la abstinencia) y características cognitivas (como la dificultad para concentrarse). La afectividad negativa implica una mayor reactividad frente a estímulos negativos, y se asocia con una mayor probabilidad de trastornos emocionales, sobre todo en las niñas.

Cuando ocurren eventos estresantes de la vida, las características cognitivas asociadas con la afectividad negativa, como los sentimientos de abandono o la pérdida de y / o baja autoestima, así como los pensamientos rumi nativos, pueden hacer que sea difícil enfrentar estos eventos, lo que aumenta la probabilidad de sufrir depresión en comparación con aquellos individuos sin estas características.

La presencia de síntomas depresivos, como anhedonia o pensamientos de muerte, aumenta significativamente el riesgo de aparición de depresión mayor en niños y adolescentes. Finalmente, los niños o adolescentes que sufren discapacidades físicas o de aprendizaje, déficit de atención, hiperactividad o trastornos de la conducta también corren un mayor riesgo.

\section{Evaluación}


La evaluación de la depresión en niños y adolescentes puede tener un propósito de diagnóstico cuando el objetivo es verificar la presencia o ausencia de criterios de diagnóstico, se puede hacer con el propósito de cuantificar la frecuencia o severidad de los síntomas, y / o el objetivo puede ser de cribado diagnóstico.

La evaluación de la depresión mayor en la infancia y la adolescencia tiene algunas características especiales, aunque comparte los objetivos de cualquier evaluación en salud mental. Por lo tanto, es importante tener en cuenta que los síntomas depresivos se expresan de manera diferente en niños que, en adultos, en parte debido a la capacidad limitada de un niño para reconocer y comunicar emociones y pensamientos negativos, sobre todo en niños más pequeños.

Por lo tanto, es importante obtener información de diferentes fuentes, además del niño, debe haber información de padres y maestros, y la evaluación debe orientarse hacia los síntomas relacionados con problemas de conducta (agresividad, rendimiento escolar, etc.) o hacia aspectos como las quejas somáticas y el retiro social, aunque también deben cuantificarse los aspectos cognitivos.

\section{Métodos de evaluación}

Existen diferentes instrumentos para evaluar la depresión en niños y adolescentes, que consisten básicamente en cuestionarios de autoinforme (auto o hetero aplicados) y entrevistas con diferentes grados de estructuración. El instrumento de evaluación seleccionado dependerá básicamente del objetivo.

Algunos instrumentos de evaluación son más generales y otros son más específicos, y algunos tienen versiones reducidas para disminuir el tiempo necesario para completarlos. Incluso se pueden usar en diferentes áreas, no solo en el área clínica. 
Carlos A. Borja-Delgado; Estefania C. Ruilova-Mera; Daisy M. Garcia-Intriago; Katerine E. Guevara-Suárez; Andrés

\section{Cuestionarios de depresión}

Uno de los métodos de evaluación más utilizados es el cuestionario. Son instrumentos cuyo objetivo es registrar sentimientos e ideas recientes y proporcionar una revisión más o menos completa de las diferentes áreas / dimensiones que podrían verse afectadas: afectiva, conductual y / o fisiológica.

Los cuestionarios de evaluación de la depresión tienen diferentes utilidades: cuantificar la intensidad de la sintomatología, establecer áreas problemáticas de comportamiento, detectar cambios sintomatológicos (monitoreo de síntomas), realizar exámenes de detección e incluso calificar el tipo de trastorno.

\section{Instrumentos basados en entrevistas}

Las entrevistas clínicas varían según el grupo de edad al que se dirige y, según el formato, pueden ser entrevistas semiestructuradas o estructuradas. Actualmente se usan ampliamente, dado que tienen la ventaja de permitir que los datos se vuelvan uniformes.

La mayoría se basa en un sistema de diagnóstico existente y, en general, ofrecen una guía para el clínico para hacer preguntas y registrar la información obtenida, lo que permite establecer un diagnóstico y estudiar la comorbilidad. Estas entrevistas requieren capacitación para ser utilizadas, y deben ser utilizadas por especialistas en salud mental.

\section{Detección de la depresión}

Diferentes estudios epidemiológicos han demostrado claramente un posible infra diagnóstico y un tratamiento inadecuado de la depresión en un alto porcentaje de niños y adolescentes. Los estudios 
realizados en los EE. UU. muestran que solo el 50\% de los adolescentes con depresión se diagnostican antes de llegar a la edad adulta y que dos de cada tres jóvenes con depresión no se detectan en la atención primaria (Zuckerbrot \& Cheung, 2007).

Por lo tanto, se ha propuesto el uso de instrumentos que son capaces de evaluar, lo que permite la detección temprana de pacientes con depresión para que puedan recibir un tratamiento adecuado o incluso permitir intervenciones preventivas en aquellos pacientes que tienen un alto riesgo de desarrollar depresión mayor.

Es importante considerar si la detección mejoraría los resultados en niños y adolescentes con depresión y si debería hacerse en forma amplia (en toda la población general, es decir, en todos los pacientes que acuden a un médico) o de manera limitada (solo en aquellos pacientes que presentan factores de riesgo).

Hay pocos datos comparativos sobre el uso de cuestionarios como instrumentos de detección en la infancia y la adolescencia. La guía NICE considera que el MFQ es uno de los cuestionarios más estudiados y más sólidos para la detección en adolescentes, aunque en España no se ha utilizado para este propósito. Con respecto a la detección de la depresión en niños, la guía NICE no recomienda el uso de ningún cuestionario de autoinforme.

El CDI ha demostrado ser un buen instrumento de detección y ha demostrado tener un alto poder de diferenciación entre los niños normales y deprimidos (Polaino \& Domenèch, 1988). Sin embargo, este cuestionario tiene algunas limitaciones, que incluyen la ausencia de elementos relacionados con los aspectos biológicos y de comportamiento, ya que se centra en los aspectos cognitivos. 
Carlos A. Borja-Delgado; Estefania C. Ruilova-Mera; Daisy M. Garcia-Intriago; Katerine E. Guevara-Suárez; Andrés

Con respecto a la persona a la que debe dirigirse el instrumento de detección para lograr los mejores resultados, existe un consenso generalizado de que las preguntas sobre los síntomas y los problemas actuales se deben hacer por separado tanto de los niños como de sus padres, y ambos grupos de respuesta se deben combinar para obtener una mejor estimación, básicamente en edades menores de 14 años. Si el instrumento de detección está dirigido solo a los padres, se detectan pocos casos de depresión (falsos negativos), mientras que, si solo se dirige a niños o adolescentes, es probable que los casos que no presentan la patología (falsos positivos) sean detectado

Otros proveedores de información potenciales podrían ser maestros, amigos o hermanos, y entre estos últimos, preferiblemente aquellos que tienen una relación más íntima, dado que podrían proporcionar información más confiable. Con respecto a la evaluación en las escuelas, en un ensayo clínico controlado aleatorio se observó que la capacitación de maestros para detectar la depresión en adolescentes no mejoró su capacidad para reconocer esta patología entre los estudiantes (Moor, y otros, 2007).

En cualquier caso, estos instrumentos de detección solo servirían para detectar una posible depresión, y sería necesario realizar un diagnóstico a través de una entrevista clínica.

Algunos autores proponen que los médicos primarios deben evaluar a los adolescentes con alto riesgo de depresión durante las citas con el médico, mientras que diferentes organismos, como el Grupo de Trabajo de Servicios Preventivos de los Estados Unidos o el Grupo de Trabajo Canadiense sobre Atención Preventiva de la Salud, consideran que la evidencia científica actual es insuficiente tanto para recomendar y no recomendando.

Debido a que no existen estudios que evalúen la efectividad de los programas de detección, generalmente no se recomienda que los programas de detección de depresión se realicen en la 
población general o en niños o adolescentes con alto riesgo. Sin embargo, se recomienda una búsqueda individual y activa de sintomatología depresiva en pacientes que tienen factores de riesgo.

\section{Conclusiones.}

Los factores sociales, como la baja educación de los padres, la baja clase ocupacional, el hecho de vivir en un hogar monoparental o tener padres desempleados, es probable que aumenten el riesgo de síntomas depresivos entre los adolescentes incluso en sociedades con un sistema de bienestar fuerte. Las niñas que viven con padres de baja educación o con un solo adulto son especialmente vulnerables. Este conocimiento debe guiar las intervenciones preventivas para reducir la desigualdad de salud mental. Se necesita trabajo continuo para reducir las desigualdades en los síntomas depresivos.

El objetivo de la investigación que sustenta este estudio fue desarrollar una comprensión de los factores que podrían influir en el desarrollo de la depresión adolescente. Este estudio proporcionó al campo de la psicología educativa una valiosa investigación en el campo de la depresión adolescente. Se obtuvo un entendimiento de la dirección y programa educativo que sirve de base para la intervención en el ámbito del adolescente la depresión debería tener.

\section{Bibliografía.}

Argimón Pallás, J., \& Jiménez Villa, J. (2000). Inferencia causal. Métodos de investigación clínica y epidemiológica.

Bragado, C., Bersabé, R., \& Carrasco, I. (1999). Factores de riesgo para los trastornos conductuales, de ansiedad, depresivos y de eliminación en niños y adolescentes.

Garber, J. (2006). Depression in Children and Adolescents. Linking Risk Research and Prevention.

Haavisto, A. (2004). Factores asociados con los síntomas depresivos en varones de 18 años: un estudio prospectivo de seguimiento de 10 años. 
Carlos A. Borja-Delgado; Estefania C. Ruilova-Mera; Daisy M. Garcia-Intriago; Katerine E. Guevara-Suárez; Andrés P. Morales-Tipan

Health., N. C. (2005). National Collaborating Centre for Mental Health. Depression in Children and Young People. Identification and management in primary, community and secondary care. Obtenido de http://www.nice.org.uk/nicemedia/pdf/cg028fullguideline.pdf

Miech, R., \& Caspi, A. (1999). Low socioeconomic status and mental disorders: a longitudinal study of selection and causation during young adulthood.

Moor, S., Maguire, A., McQueen, H., Wells, E., Elton, R., \& Wrate, R. (2007). Improving the recognition of depression in adolescence: can we teach the teachers?

Polaino, A., \& Domenèch, E. (1988). La depresión en los niños españoles.

Reiss, F. (2013). Desigualdades socioeconómicas y problemas de salud mental en niños y adolescentes.

Vos, T., Flaxman, A., Naghavi, M., \& Lozano, R. (2012). Years lived with disability (YLDs) for 1160 sequelae of 289 diseases and injuries 1990-2010: a systematic analysis for the Global Burden of Disease Study 2010.

Zuckerbrot, R., \& Cheung, A. (2007). Guidelines for Adolescent Depression in Primary Care. 